Tome 44, no 13. - Avril 1937.

\title{
Scorpions, Solifuges et Opilions d'Angola
}

\author{
par \\ Albert MONARD \\ $D^{r}$ ès-sciences, Directeur du Musée d'Histoire naturelle \\ de La Chaux-de-Fonds.
}

\section{SCORPIONIDES.}

\section{INTRODUCTION.}

Les Scorpions sont médiocrement répandus en Angola; en quelques endroits même, ils paraissent ne pas exister ou du moins être très rares, à en juger par les indigènes qui n'ont pas reconnu les dessins que je leur montrais; en d'autres, je n'ai pu recueillir que quelques exemplaires; c'est seulement à Mupanda que la chasse a été vraiment fructueuse et que j'ai trouvé de nombreux individus en plusieurs espèces.

Cette inégalité dans la distribution et la fréquence de ces animaux doit être attribuée à la constitution du sol; les Scorpions affectionnent en effet les endroits secs, sablonneux ou pierreux. Or, dans le centre de l'Angola, la saison des pluies est sévère et le sol de nature latéritique ou argileuse, conditions défavorables au développement de leur faune. Mais dans le Sud, où le sable apparaît (Chimporo, Mupanda, etc.) les Scorpions deviennent plus abondants.

Les Scorpions habitent d'ordinaire les vieux murs, les pierres, les ruines où ils trouvent des cachettes pour se dissimuler. Or, en Angola, cet habitat n'existe pas; les constructions indigènes sont faites en terre battue, ne donnent pas de ruines durables et disparaissent même complètement. Les pierres sont rares dans les pays

- Rev. Suisse de Zool., T. 44, 1937. 
à latérite et n'existent guère que dans les montagnes. Les Scorpions ont trouvé ailleurs leurs cachettes et ils ne se rencontrent que sous les écorces des arbres tombés; cet habitat est si fréquent que les Ngangelas qui n'ont qu'un nom pour désigner les Scorpions et les Scolopendres, "Inyé", mais qui distinguent fort bien les deux bêtes, appellent les premiers "Scorpions des arbres" (Ynyé ya miti) et les seconds "Scorpions de terre " (Inye ya hantsi).

Au Kuanyama, où ces animaux abondent, ils ont trouvé d'autres cachettes. Le pays est sablonneux et si peuplé qu'il n'y a pas d'arbres tombés; aussi les Scorpions se réfugient dans une espèce d'aloès très abondante dans le pays et se dissimulent dans les aisselles des feuilles inférieures. Il suffit donc de les écarter légèrement pour trouver souvent, à leur base, un ou deux Scorpions cachés. Cet habitat est très régulier et les nombreux Scorpions rapportés de ce pays ont tous été trouvés dans les aloès, à moins qu'ils ne se soient mis en chasse.

Très peu de choses ont été écrites sur les Scorpions d'Angola. Pососк (1896) cite Uroplectes occidentalis, sans indication de localité. On trouve encore Archisometrus asper Poc. dans Kraepelin. Le mémoire de John Hewitt "A Survey of the Scorpion Fauna of South Africa" (Transactions of the Royal Society of South Africa, vol. VI, 1917, pp. 89-192) ne mentionne que trois fois cette colonie, à propos de Parabuthus villosus, Uroplectes planimanus et Hadogenes tityrus. Dans les "Résultats de la Mission Rohan Chabot ", t. IV, fasc. 3 (1925) Louis FAGE ne trouve que deux espèces: Uroplectes planimanus et Parabuthus brevimanus. Enfin existe notre propre mémoire sur les Scorpions de notre premier voyage, paru dans le Bulletin de la Société neuchâteloise des Sciences naturelles, t. 54, 1929, pp. 37 à 43, avec quatre espèces, que nous reprendrons dans le cours de ce travail. C'est tout ce que nous avons trouvé concernant l'Angola:

Voici les stations d'où nous avons rapporté des Scorpions:

Premier voyage (1928-29):

Katumbela: Buthus angolensis.

Rio Mbalé: Uroplectes planimanus.

Kakindo: Uroplectes planimanus, Parabuthus raudus. Chimporo: Parabuthus raudus, Uroplectes otjimbinguensis. Tumbolé: Uroplectes planimanus. 
Deuxième voyage (1932-33).

Kuvangu: Uroplectes fischeri, planimanus.

Mukoti: Uroplectes fischeri.

Kului: Uroplectes vittatus.

Indungu: Uroplectes planimanus.

Kampulu-Kambisa: Uroplectes carinatus, otjimbinguensis.

Kuvelaï: Parabuthus brevimanus, Uroplectes carinatus, planimanus.

Tyihumbwé: Opisthophthalmus lundensis, Uroplectes occidentalis. Ebanga: Uroplectes planimanus.

Kaamba: Uroplectes planimanus, otjimbinguensis.

Mupa: Uroplectes planimanus, otjimbinguensis.

Mupanda: Parabuthus kuanyamarum, granulatus, raudus, Buthus angolensis, Uroplectes carinatus, planimanus.

Dombondola: Parabuthus brevimanus, granulatus.

Nous avons rapporté, au total, douze formes de Scorpions, répartis en deux familles et quatre genres:

1. Sternum pentagonal; à la base du dernier article des pattes un seul éperon externe dans la membrane articulaire. Yeux placés en arrière du milieu du céphalothorax: Famille des Scorpionidae, g. Opisthophthalmus.

- Sternum triangulaire; à la base du dernier article des pattes, 1 ou 2 éperons internes et externes: Famille des Buthid ae, 2.

2. Doigt fixe des chélicères avec 2 dents à son bord inférieur 3 .

- Doigt fixe des chélicères sans dent à son bord inférieur: Uroplectes.

3. Trois carènes dorsales aux tergites abdominaux: Buthus.

-- Une carène dorsale aux tergites abdominaux: Parabuthus.

\section{Buthid Ae.}

Buthus angolensis Monard.

Buthus angolensis, Monard, 1929.

Nous avons décrit cette espèce d'après un seul exemplaire mâle (et non femelle) provenant apparemment de Katumbela; au deuxième voyage, pendant notre séjour à Mupanda, les indigènes nous ont apporté un grand nombre d'individus appartenant à cette 
espèce, si bien que nous pouvons en donner maintenant une description comparée.

Matériel: 42 femelles et 23 mâles de toutes grandeurs.

La coloration est peu variable et permet à première vue de reconnaître l'espèce. Mâles et femelles ont les faces supérieures d'un jaune assez foncé où tranchent les dessins noirs du champ qui s'étend des yeux médians au bord frontal, des carènes du céphalothorax et du bord latéral de ce dernier, partie antérieure. Les chélicères sont d'un jaune vif et brillant avec les mors des pinces bruns. Sur l'abdomen s'étendent cinq rangées de taches noires, celles du milieu confluentes en ligne, les latérales plus disjointes. Le postabdomen est jaune brunâtre, les carènes renforcées de ton; par dessous la couleur se fonce d'article en article, les trois derniers brun foncé. La face inférieure du tronc est plus claire; les quatre carènes du dernier segment sont brunes, les peignes très clairs, les pattes un peu rembrunies à leur côté supérieur, surtout les pinces. Les jeunes exemplaires ont les teintes générales plus claires où tranchent plus nettement les taches noires; les grands exemplaires sont de teinte plus foncée.

Céphalothorax: les crêtes sourcilières sont tuberculeuses, arquées, et se rejoignent assez nettement sur le front; les crêtes latérales médianes sont confuses, tuberculeuses; les crêtes médianes postoculaires sont parallèles, un peu plus distantes que les yeux et rejoignent plus ou moins nettement les crêtes postérieures, qui sont plus écartées que les postoculaires. La surface entière est fortement granuleuse, front compris.

Les tergites sont fortement granuleux; les trois carènes sont bien nettes; le dernier tergite est orné d'une crête médiane, triangulaire, plus courte que le segment et de deux paires de carènes latérales, rapprochées en arrière, courbées.

Les sternites sont lisses au milieu, finement granuleux en avant et autour de la protubérance des stigmates; ceux des femelles sont plus lisses encore. Le dernier sternite est muni de quatre crêtes granuleuses, les médianes aboutissant au bord postérieur, les latérales plus courtes; l'avant-dernier sternite présente aussi quatre crêtes peu marquées, surtout les médianes, lisses chez les femelles, bien marquées et légèrement granuleuses chez les mâles. Les sternites de ces derniers sont en général plus granuleux dans l'ensemble que ceux des femelles. 
Postabdomen: les quatre premiers articles sont semblables, à dix carènes bien marquées et fortement granuleuses; les surfaces ventrales, latérales et supérieures sont granuleuses. Le cinquième article a cinq carènes tuberculeuses et des surfaces rudes à l'exception de la fosse où se replie la vésicule. Celle-ci est fortement tuberculeuse, en cinq rangées.

Doigt mobile du maxillipède: il y a douze rangées de granules, chacune d'elles débutant par deux points plus gros et plus obliques; en outre, un granule interne vers le milieu de chaque ligne.

Peigne: 24 à 25 dents chez les mâles, 17 à 20 chez les femelles.

Discussion: cette espèce fait partie du groupe hottentota et se rapproche des espèces trilineatus Ptrs, conspersus Thor et arenaceus Purc. Elle représente vraisemblablement une forme plus occidentale de ce groupe austral. Voici un tableau permettant de se rendre compte des différences:

\begin{tabular}{|c|c|c|c|c|}
\hline & trilineatus & conspersus & arenaceus & angolensis \\
\hline $\begin{array}{l}\text { Points du } \\
\text { maxillipède }\end{array}$ & 13 à 14 séries & 12 séries & 10 à 11 séries & 12 séries \\
\hline Peigne & $\begin{array}{l}25 \text { dents } \\
(15-17)\end{array}$ & 14 dents & 17 à 18 dents & $\begin{array}{l}17 \text { à } 20 \text { fem. } \\
24 \text { à } 25 \mathrm{~m} \text {. }\end{array}$ \\
\hline Sternites & $\begin{array}{l}\text { lisses au mi- } \\
\quad \text { lieu }\end{array}$ & $\begin{array}{l}\text { lisses au mi- } \\
\quad \text { lieu }\end{array}$ & granuleux & $\begin{array}{l}\text { lisses au mi- } \\
\text { Jieu }\end{array}$ \\
\hline $\begin{array}{l}\text { Sternite IV, } \\
\text { crêtes }\end{array}$ & 1 p. latérales & $\begin{array}{l}4 \text { subgranu- } \\
\text { leuses }\end{array}$ & 4 granuleuses & $\begin{array}{l}4 \text { lisses (fem.) } \\
4 \text { granuleuses } \\
\text { (mâles) }\end{array}$ \\
\hline
\end{tabular}

On voit que c'est de trilineatus que notre espèce est la plus proche. Elle en diffère néanmoins:

$1^{\circ}$ par les crêtes préoculaires qui sont fortement granuleuses et prolongées jusqu'au front ("glatt oder etwas kerbig bis feinkörnig", dans trilineatus, d'après KraEpelin);

$2^{\circ}$ par les carènes des tergites, nettement granuleuses et à grains séparés ("etwas leistenartig verschmolzen» dans trilineatus);

$3^{0}$ par les carènes inférieures du premier segment postabdominal, fortement tuberculeuses ("glatt oder kerbig") dans trilineatus);

$4^{0}$ par les carènes dorsales des segments III et IV qui sont semblables aux autres ("stark sägezähnig-zackig » dans trilineatus); 
$5^{\circ}$ par l'absence des deux crêtes tuberculeuses sur la main du maxillipède;

$6^{\circ}$ par la main du maxillipède pas plus large que le tibia chez le mâle ("viel dicker » dans trilineatus);

$7^{\circ}$ par les doigts de la pince une fois et demie au moins plus longs que la main (une fois et un tiers dans trilineatus).

Ajoutons cependant une remarque de Kraepelin "eine sehr variable Art, die vielleicht in mehrere selbständige Formen $\mathrm{zu}$ zerlegen ist ". Notre espèce paraît être une de ces formes, mais bien nettement délimitée et d'habitat géographique plus occidental. Trilineatus est une espèce plutôt orientale; le type est de Tette sur le Zambèze, et d'après Hewitт elle existe dans le district Feira, près de la rivière Loangwa, affluent du Zambèze, dans le Nord-Est de la Rhodésia, dans l'ancien German East africain et au Transvaal. Ce sont là des localités assez éloignées du Kuanyama et de Katumbela où nous avons trouvé notre espèce, pour qu'il se soit formé ici une forme bien définie.

\section{Parabuthus Pocock.}

Ce genre difficile est représenté dans nos collections par quatre espèces dont voici la clef:

1. Côtés et surface inférieure du IVe article postabdominal lisses ou granuleux, mais sans crête granuleuse distincte. Front granuleux.

brevimanus.

- IVe article avec deux crêtes granuleuses (lat. inf.). Front lisse. kuanyamarum.

— IVe article avec huit carènes, les latérales intermédiaires rudimentaires sauf en arrière.

granulatus.

- IVe article avec dix crêtes, les latérales intermédiaires bien développées. Crêtes dorsales du V interrompues au milieu, mais alors doublées par une crête dorsale accessoire, composée de tubercules pas plus hauts que larges. raudus.

\section{Parabuthus brevimanus Thorell.}

Une femelle de Dombondola; un jeune du Kuvelaï.

Ces deux exemplaires correspondent dans tous leurs détails à la description de Hewitт. Notamment, les crêtes inférieures et 
inféro-latérales des $\mathrm{II}^{\mathrm{e}}$ et $\mathrm{III}^{\mathrm{e}}$ articles du postabdomen, par leur jonction, sont très caractéristiques. Les crètes latérales du Ve article, peu marquées en avant, se terminent en arrière par trois lobes carrés proéminents. La couleur est jaune clair, un peu verdâtre sur l'abdomen.

C'est une espèce du Sud-Ouest africain, citée dans le Great Bushmanland, le Sud-Ouest, le Betchuana et jusqu'au Congo. La mission Rohan-Chabot l'a ramenée de la rivière Kuatiri (affluent du Kuvangu).

\section{Parabuthus granulatus (H. \& E.).}

Dombondola: une femelle de $66^{\mathrm{mm}}$ de longueur.

Mupanda: 7 femelles, pour la plupart gonflées d'œufs et prêtes à pondre (juillet).

L'espèce habite les aisselles des feuilles inférieures d'Aloès.

La couleur est jaune, le trone plus foncé. Parfois le postabdomen est brunâtre ou même noirâtre. Il y a de 27 à 29 dents aux peignes. Nos exemplaires sont bien typiques dans tous leurs détails; la couleur générale paraît cependant plus claire.

La distribution de cette espèce comprend: le Namaqualand et le Kalahari; elle ne s'étend pas loin vers l'Est et le Sud. Nos deux stations, situées à la frontière sud de l'Angola, étendent, loin vers le Nord, l'aire distributive de cette espèce.

\section{Parabuthus raudus Simon.}

Mupanda: 5 femelles et 2 mâles.

Chimporo: 22 exemplaires, mâles et femelles.

Kakindo: 1 exemplaire.

Il s'agit ici de la forme redécrite par Kraepelin, puis par Hewitt sous le nom de raudus; comme villosus est cité en Angola, nous avons soigneusement confronté les caractères des deux espèces. Mais les carènes dorsales du Ve article du postabdomen sont franchement interrompues au milieu et ne reprennent que confusément en arrière, caractère de raudus, tandis qu'elles sont nettes tout au long dans villosus; en outre, les carènes médianes du premier article sont lisses, les doigts de la pince plutôt courts et la villosité moindre.

L'espèce est citée au Kalahari (Simon), dans le Betchuanaland (Kraepelin), puis dans le Great Namaqualand (Hewitt). Nos 
stations montrent qu'elle s'étend jusqu'à la limite supérieure du régime kalaharien.

\section{Parabuthus kuanyamarum nov. sp.}

Matériel: 3 femelles et 4 mâles venant de Mupanda, juillet 1933. Longueur de la plus grande femelle: $42 \mathrm{~mm}$.

Couleur entièrement jaune, rougeâtre sur le postabdomen, un peu plus foncée sur le corps, plus claire sur les pattes; aiguillon brun.

Céphalothorax: aire interoculaire lisse, coupée par un sillon lisse aussi; surfaces finement chagrinées sur les côtés et derrière les yeux, au bord postérieur du céphalothorax; deux protubérances lisses derrière les yeux, séparées par un sillon finement chagriné.

Abdomen: segments lisses sur les côtés et en avant, chagrinés dans le voisinage de la carène et en arrière; carènes lisses. Dernier segment à quatre carènes finement tuberculeuses; surface située entre les carènes médianes, finement chagrinée; surfaces latérales plus grossièrement tuberculeuses. Sternites lisses, le dernier à quatre carènes indistinctes.

Postabdomen, article I: les quatre carènes inférieures lisses, les six autres un peu granuleuses. Surface supérieure chagrinée jusqu'au bord postérieur de l'article, assez large, finissant brusquement en escalier du côté antérieur. Surfaces intercarénales lisses dessous, granuleuses seulement en arrière sur les côtés.

Article II : carènes inférieures lisses, les supérieures granuleuses. Surfaces intercarénales lisses, les latérales granuleuses postérieurement, comme au premier article. Surface chagrinée supérieure étroite, occupant le fond d'un sillon et s'étendant jusqu'au bord postérieur de l'article.

Article III: les quatre carènes inférieures granuleuses, les latérales, les médianes et les dorsales presque effacées. Surface inférieure (entre les carènes médianes) un peu granuleuse, les autres lisses à l'exception des latérales un peu granuleuses postérieurement.

Article IV : carènes effacées à l'exception des deux latérales inférieures nettes et granuleuses (différence d'avec brevimanus et calvus). Surfaces granuleuses dessous et sur les côtés, postérieurement, comme à l'article précédent.

Article V: les deux carènes latérales inférieures seules marquées et Cortement granuleuses, surtout postérieurement; surfaces infé- 
rieures fortement granuleuses, de même que celles qui bordent supérieurement la carène subsistante. Surfaces supérieures lisses.

Vésicule: deux sillons ventraux; surfaces inférieures et latérales granuleuses. Villosité modérée.

Pince: main étroite, à peine plus large que le brachium; doigts courts, le mobile à peine plus long que la main (différence d'avec calous). Doigt mobile avec 9 à 10 rangées de granules, les internes se mettant en triple avec les deux premiers de chaque série.

Peigne de la femelle comptant 22 à 23 dents, la première courte et très fortement élargie; celui du mâle avec 25 à 26 dents, la première normale.

Discussion: cette nouvelle forme de Scorpion s'apparente avec brevimanus Thor et calvus Purc. Mais elle en diffère par l'aire interoculaire lisse, comme dans laevifrons Simon et par la présence de deux carènes au quatrième article du postabdomen. Ce caractère ne se trouve pas encore réalisé dans le genre; les espèces brevimanus et calous n'ont aucune carène à cet article, les autres en ont huit ou dix. Il y a en outre des différences de détail portant sur l'aspect des différentes surfaces, du peigne, de la pince et des carènes du postabdomen.

\section{Uroplectes Peters.}

Nous avons rapporté six espèces de ce genre, dont l'une présente trois formes:

1. Tergites munis de trois carènes dorsales granuleuses, les latérales plus courtes. Au doigt mobile de la pince, les granules internes sont isolés, disposés vers l'extrémité de chaque série, celles-ci débutant par deux forts granules obliques; pas de dent sous l'aiguillon.

- Tergites munis seulement d'une carène médiane; postabdomen sans carène distincte; granules internes isolés, situés vers le milieu de chaque rangée, au moins à la base du doigt. Une dent sous l'aiguillon.

2. Main de la pince aplatie et élargie, beaucoup plus large que le brachium, à côté interne plus ou moins tranchant; dent basale du peigne de la femelle allongée et falciforme.

planimanus.

- Main étroite, à peine plus large que le brachium, arrondie. Dent basale du peigne de la femelle variable. carinatus. 
3. Tronc avec bande noire médiane. Granules externes (du doigt mobile de la pince) disposés par paires, granules internes toujours isolés.

otjimbinguensis.

- Tronc avec une paire de bandes sombres, la carène claire (rarement sombre au milieu de l'espace clair), granules externes disposés par deux ou trois; granules internes isolés ou appairés.

4. Postabdomen granuleux à la face ventrale, surtout aux segments IV et $\mathrm{V}$; carènes dorsales un peu indiquées aux segments I et II.

vittatus.

- Postabdomen à faces ventrales ponctuées de petites fossettes; carènes dorsales toutes effacées ou remplacées par des ponctuations, ou marquées seulement par la dent terminale.

5. Au dernier tiers du doigt mobile de la pince, les granules terminaux de chaque série sont plus gros et très nettement séparés des précédents, appairés avec les granules internes.

occidentalis.

- Ces granules à peine plus grands et à peine séparés des précédents, indistinctement appairés avec les internes.

fischeri.

Uroplectes planimanus Karsch.

L'espèce varie notablement selon les régions; du reste les auteurs ne sont pas d'accord sur ses relations avec $U$. carinatus Pocock et en donnent des descriptions quelque peu divergentes. Ainsi Pocock dit que les carènes supérieures des segments I et II du postabdomen sont faiblement granuleuses, tandis que Kraepelin indique: "Cd. im I. und II. Segm. zehnkielig, mit glatten Kiele ", ne faisant ainsi aucune distinction entre les carènes ventrales et les dorsales. Au IVe article, les carènes latérales intermédiaires sont nulles dans planimanus, bien marquées dans carinatus selon Pococк et Kraepelin; mais ce caractère doit être abandonné selon Hewitt. $\mathrm{Au} \mathrm{Ve}$ article, Kraepelin indique trois carènes nettes. Le nombre des dents des peignes et supérieur à 30 selon Pocock, de 28 à 30 selon Kraepelin (1899), de 22 à 29 chez le mâle et de 20 à $27 \mathrm{chez}$ la femelle, selon Hewitt.

Notre matériel, abondant, montre trois formes de ce Scorpion. 
A. La première est celle que nous avons nommée, en 1929, Uroplectes ngangelarum; nous en possédons de nombreux exemplaires, tous bien semblables, venant de Vila da Ponte, rio Mbalé, Kakindo, Tumbolé, Indungu. En raison des divergences citées plus haut et en comparaison avec les deux autres formes, nous ne pouvons maintenir ce Scorpion au rang d'espèce, et il doit être rangé parmi les formes de planimanus. Cette variété est caractérisée: $1^{0}$ par les carènes dorsales des segments I et II, nettement et assez grossièrement granuleuses, tandis qu'elles sont faiblement granuleuses (Рососк) ou même lisses (KrAEPELIN) dans planimanus; $2^{\circ}$ par la présence au IVe article de quatre carènes seulement, les autres effacées, parfois presque complètement, ailleurs légèrement indiquées (huit carènes dans planimanus selon Kraepelin); $3^{\circ}$ par l'absence de la carène médiane inférieure au Ve segment (présente dans $p l$. selon Kraepelin). Les peignes ne possèdent qu'un nombre réduit de dents, ordinairement 22 ou 23 chez la femelle, 24 ou 25 chez le mâle. Ces nombres sont inférieurs à ceux qu'indiquent Pосоcк et Kraepelin, mais sont contenus dans les limites assignées par Hewits. La coloration est aussi différente: le bord frontal est toujours foncé, le postabdomen marqué par quatre lignes fines sur chaque article, suivant les carènes inférieures (2 lignes selon Kraepelin), le postabdomen n'est pas obscurci à son extrémité.

B. La deuxième forme, en plusieurs exemplaires mâles et femelles, vient de Mupanda et pourrait être nommée Uroplectes planimanus kuanyamarum. Les carènes du postabdomen sont celles de ngangelarum, mais la coloration d'ensemble est beaucoup plus claire et rappelle celle de planimanus. Le postabdomen, les pinces et les pattes sont d'un jaune très clair, sans aucune trace de foncé, sauf à l'aiguillon brun. En outre, chez les mâles, le postabdomen est proportionnellement plus grêle et plus allongé, surtout au IVe et Ve articles, presque égaux dans quelques exemplaires, le premier toujours plus court que le second dans ngangelarum. Chez la femelle, la dent basale du peigne est plus allongée et plus falciforme que dans cette dernière.

Des individus pris à Mupanda et à Kamba sont intermédiaires entre les deux variétés.

C. Un seul exemplaire, venant d'Ebanga, est extrêmement différent par sa couleur. Le tronc et le postabdomen sont noirâtres, la vésicule jaune clair, l'aiguillon brun. Les pattes sont de la même 
couleur que le dos à leur face supérieure, plus claires à leur extrémité et à leur face inférieure. Les carènes sont celles de ngangelarum.

La présence de ce Scorpion est signalée en Angola par Hewitt (un mâle provenant du $\mathrm{D}^{\mathrm{r}}$ Ansorge) puis par Louis FAGE (entre le Kuvangu et Mukuva). C'est certainement l'espèce la plus répandue dans le centre de la colonie.

Uroplectes planimanus est une espèce du Sud africain (Mashona et Windhoeck). Elle est en outre citée par HewitT dans les régions suivantes: Great Namaland et Damaraland, Mashona, Tette, chutes Victoria, Rhodésia du N.-E., Betchuanaland, Tati, Eastcourt, Transvaal. Nos stations, les plus équatoriales connues, agrandissent vers le centre africain l'aire distributive de l'espèce.

\section{Uroplectes carinatus Pocock.}

Nous attribuons à cette espèce, avec doute cependant, six exemplaires venant de Mupanda (deux mâles, une femelle), du Kuvelaï (un jeune mâle), de Kambisa (deux femelles). D'après Poсоск cette espèce différerait de planimanus par les carènes inférieures du premier segment postabdominal fortement granuleuses, par la carène latérale intermédiaire forte au troisième segment, distincte au quatrième, et par la main du mâle normalement arrondie. Ces caractères ont été repris par Kraepelin; mais Hewitt (1917) dit que les carènes inférieures peuvent être lisses et que les caractères tirés de la conformation des carènes ne peuvent être utilisés: "The only satisfactory characters available for distinguishing the two species are the shape of the hands and the character of the basal pectinal tooth in the female ".

Nos exemplaires, tous de petite taille, se rapprochent étrangement de planimanus ngangelarum; mais ils ont la main non élargie, à peine plus large que le tibia et fort différente de celle de planimanus. Quant à la dent basale du peigne de la femelle, elle est un peu plus longue que les autres et légèrement élargie. C'est donc uniquement sur la forme de la main de la pince que nous pouvons nous baser pour séparer ces exemplaires de planimanus.

Purcell, Kraepelin et Hewitt ont distingué plusieurs variétés dans cette espèce: c'est de mediostriatus Kr. du Kalahari, que nos individus sont les plus proches.

Peut-être aussi pourrions-nous envisager ces exemplaires comme 
appartenant à une race nouvelle de carinatus; mais ils sont trop jeunes pour que nous puissions les décrire avec certitude.

$U$. carinatus est répandu surtout dans la région occidentale du Sud africain, chaque variété occupant une aire particulière. Mais il n'était pas encore signalé dans des stations aussi élevées vers le Nord.

\section{Uroplectes otjimbinguensis Karsch. v. massacarum n. v.}

Dans notre mémoire sur les Scorpions d'Angola, nous avons cité cette espèce d'après un mâle du Chimporo; ce mâle n'avait sous l'aiguillon que quelques tubercules, le dernier un peu plus gros, mais ne pouvant passer pour une dent. A notre deuxième voyage, nous avons rapporté quatre exemplaires de ce Scorpion, semblables dans tous leurs détails à celui du Chimporo, mais ayant sous l'aiguillon une dent triangulaire nette. Nous pensons donc, en s'appuyant sur ce caractère, distinguer nos exemplaires du type d'otjimbinguensis et créer pour eux la variété massacarum.

Matériel: 1 femelle de Kampulu (type), 2 mâles de Kâmba, 1 jeune de Mupa.

La couleur est jaune-clair; les bords du céphalothorax et des tergites sont noirs; une bande noire médiane, élargie en avant des yeux et formant un triangle, occupe l'espace des carènes abdominales. Le postabdomen est un peu assombri à son extrémité; une raie médiane inférieure orne les segments II à IV; moitié proximale du segment $\mathrm{V}$ aussi brunâtre. Vésicule étroite, non marqué de noir; une petite dent triangulaire sous l'aiguillon. Postabdomen hérissé de longues soies éparses. Pattes et maxillipèdes clairs; doigts de la pince un peu assombris.

Surfaces des tergites grossièrement granuleuses à l'exception d'une double tache antérieure de la carène médiane et du bord antérieur. Pas de carène latérale. Sternites lisses avec chacun deux sillons latéraux, plus effacés au dernier.

Doigt mobile de la pince: 10 rangées de granules, les deux premiers de chaque série plus gros et disposés obliquement, surtout distalement. Un seul granule interne situé d'abord vers le milieu de la série correspondante, puis vers l'extrémité des dernières rangées. Ce granule est toujours isolé et ne s'appaire pas avec le distal voisin; celui-ci semblable aux autres granules. 
Postabdomen: carènes absentes à tous les segments. Le premier article présente toutefois une ébauche de crète dorsale tuberculeuse; la face ventrale de tous les articles présente des ponctuations d'où naissent les poils. Faces supérieures des segments II à V avec des ponctuations plus ou moins rangées en lignes selon les carènes.

U. otjimbinguensis est d'après Hewitt une variété de coloration de vittatus; mais celui-ci, d'après Kraepelin, aurait les surfaces du postabdomen " nicht grubig nadelstichig » (ce qui est le cas pour nos exemplaires) et " höchstens runzelig oder fein gekörnt ». D'autre part, $U$. chubbi Hirst a le sillon médian supérieur des articles II à IV du postabdomen garni de granules, ce qui n'est pas le cas de nos exemplaires.

Les rapports entre ces formes s'établiraient ainsi:

1. Surfaces inférieures du postabdomen plus ou moins granuleuses dessous, non ponctuées; un tubercule sous l'aiguillon; une bande centrale claire bordée de foncé. vittatus.

- Surfaces inférieures ponctuées; une bande médiane noire sur le trone.

2. Pas de tubercule sous l'aiguillon; onze rangées de granules au doigt mobile de la pince. otjimbinguensis.

- Un tubercule sous l'aiguillon; dix rangées de granules. otj. v. massacarum.

$U$. otjimbinguensis est une espèce rare signalée seulement à Otjimbingue, dans le Damara et le Herreroland. L'aire distributive s'étend donc encore vers le Nord puisqu'elle comprend l'Angola, région frontière.

Uroplectes occidentalis Simon.

Matériel: 3 mâles, 1 femelle, venant de la Lunda (Tyihumbwé supérieur, dans la région de Dala).

Cette espèce appartient au groupe difficile des formes d'Uroplectes où les carènes postabdominales sont absentes ou marquées seulement par un tubercule final. Elle se distingue des autres, d'abord par les surfaces ponctuées du postabdomen, puis surtout par 
la disposition des granules du doigt mobile de la pince. Ceux-ci sont disposés en douze séries, et, à l'extrémité, le granule final de chaque petite série est éloigné de ses voisins et distinctement plus gros qu'eux; en outre il s'appaire nettement avec le granule interne.

Ces caractères sont réalisés dans cette petite série de Scorpions de la Lunda. La coloration est toutefois un peu différente de celle décrite pour cette espèce: la couleur générale est assez sombre. Le céphalothorax est marbré de noirâtre, le front en avant et l'éminence oculaire noirs aussi. Les tergites ont des taches latérales marbrées de noir, la carène médiane sombre, mais les bords latéraux clairs; la surface inférieure du postabdomen est marquée de cinq fines lignes noires, dont les deux paires latérales s'unissent en tache distalement. Le segment $\mathrm{V}$ est noir en arrière, la vésicule brun-rouge avec l'aiguillon noir.

$U$. occidentalis n'est pas signalé dans l'œuvre de Hewitt. Il représente en effet, dans la faune des Scorpions d'Angola, un élément équatorial qui tranche avec les autres espèces, d'origine australe. L'espèce est signalée depuis Sierra Leone jusqu'au Congo, de la Somalie au Natal; en outre, dans les îles de la Sonde et en Cochinchine; Pocock la cite déjà de l'Angola.

\section{Uroplectes vittatus Thor.}

Nous attribuons à cette espèce un seul individu femelle recueilli sur les bords du Kului, mesurant $35^{\mathrm{mm}}$ et ayant 18-19 dents au peigne, la première élargie.

L'abdomen est verdâtre avec une paire de bandes noires, un triangle noir sur le front et deux lignes de même couleur sur la carapace. Les pattes montrent des lignes noires, sur le fond clair; les pinces sont noires avec l'extrémité des doigts clairs; une ligne foncée sous le postabdomen; vésicule jaune foncé. Chélicères réticulés de sombre.

Les granulations du doigt mobile de la pince sont semblables à celles de fischeri, mais la surface ventrale des segments IV et V du postabdomen est nettement granuleuse et aux deux premiers segments les carènes dorsales et dorsolatérales sont indiquées et granuleuses. C'est sur ces caractères que nous nous sommes basés 
pour séparer cet individu des deux exemplaires de fischeri que nous avons rapportés et qui lui ressemblent beaucoup.

L'espèce est, selon Kraepelin, répandue en Caffrerie, au Transvaal et dans la baie de Delagoa; Hewitr ajoute le Betchuanaland, le Damara, l'Ovambo et le Kalahari Ouest. C'est encore un élément. austral de la faune des Scorpionides d'Angola.

\section{Uroplectes fischeri Karsch.}

Deux spécimens: une femelle mesurant $53 \mathrm{~mm}$ venant du Kuvangu et un mâle de même longueur venant du Mukoti. Les peignes comptent dans les deux exemplaires 19 et 20 dents, la basale de la femelle très élargie.

La couleur est d'un jaune-rouge foncé. Une tache triangulaire préoculaire, deux raies obliques sur la carapace, une paire de taches sur chaque tergite, une large raie inférieure au postabdomen et des taches postérieures latérales à chaque segment, noirs. Cette coloration est celle de fischeri, vittatus et occidentalis, à peu de chose près. Les segments du postabdomen sont longuement velus. Une dent triangulaire sous l'aiguillon.

Les surfaces inférieures du postabdomen sont lisses; à un fort grossissement, elles apparaissent finement réticulées et ponctuées; en outre, de préférence sur les places des carènes, de grandes fossettes, plus rares et plus petites sur la vésicule. Les carènes dorsales des cinq segments sont marquées par quelques ponctuations et par une dent terminale. Les surfaces dorsales, concaves en section transversale, convexes en section sagittale, sont lisses et ne présentent que la très fine ponctuation indiquée plus haut, plus rare cependant qu'aux surfaces inférieures.

Au doigt mobile de la pince, à chaque petite série de granules, le dernier point est un peu plus grand et un peu séparé des précédents; il montre une tendance à former des paires avec le granule interne, mais cette disposition est beaucoup moins accusée que dans occidentalis.

$U$. fischeri est une espèce plutôt orientale; nos individus pourraient former une race valable de cette espèce, différant du type par la présence de douze séries de granules au doigt mobile de la pince (onze dans fischeri) et par la double ponctuation décrite plus haut, qui n'est pas signalée par les auteurs. 
Scorpionid ae.

Opisthophthalmus lundensis nov. sp.

5 femelles adultes, 1 mâle et 2 jeunes; le plus grand exemplaire mesure $86 \mathrm{~mm}$.

Couleur d'un brun rougeâtre ou verdâtre; céphalothorax plus jaune et plus brillant, les tergites grisâtres et mats en avant, plus bruns et brillants en arrière chez la femelle, mats partout chez le mâle. Postabdomen de couleur peu différente, les surfaces supérieures parfois plus claires. Vésicule plus claire à aiguillon brun. Pattes jaunes, les extrémités des articles marquées d'une petite tache foncée. Pinces et chélicères jaunes, à doigts bruns. Sternites jaunes, brillants.

Céphalothorax: front échancré au milieu; sillon préoculaire lisse, non fourchu, mais seulement élargi à son extrémité antérieure. Sillon interoculaire lisse, non bordé de tubercules. Yeux centraux reculés au delà du milieu du céphalothorax ( $8^{\mathrm{mm}}$ et $6^{\mathrm{mm}}$ ) aux quatre septièmes environ. Espace compris entre les yeux et le front lisse, mais côtés granuleux. En arrière une profonde impression centrale et deux latérales obliques puis franchement transversales vers l'avant. Tergites III à IV munis d'une carène médiane, le dernier sans carène, granuleux par places. Sternites lisses, le dernier sans carène.

Postabdomen: article I: carènes à peu près effacées, lisses, sauf les dorsales et les dorsolatérales qui sont indistinctement indiquées par quelques granules.

Articles II et III : les carènes sont pareilles, mais un peu mieux marquées.

Articles IV: carènes encore mieux marquées, les inférieures devenant un peu tuberculeuses, les dorsales plus nettes.

Article V: carène inférieure irrégulière, fortement tuberculeuse, les latérales inférieures plus régulières, très granuleuses aussi.

Vésicule: deux sillons lisses, limitant trois champs granuleux.

Surfaces supérieures toutes lisses, les inférieures et latérales lisses aussi, sauf la cinquième qui est granuleuse.

- Villosité développée, à soies longues. 
Palpe: Main courte et large, pas plus longue que large, à surface supérieure arrondie, à bord interne assez tranchant, à surface supérieure finement granuleuse, les granules placés en lignes réticulées. Carène du doigt peu marquée. Mors du doigt immobile formé de cinq rangées concaves de granules, situées sur le même plan et séparées par des tubercules plus gros; quelques granules hors série près de l'extrémité. Surfaces inférieures lisses, avec quelques granules épars. - Coxa: surface touchant le lobe maxillaire de la première patte lisse. Fémur quadrangulaire, les quatre carènes granuleuses, les surfaces antérieure et supérieure tuberculeuses. Tibia à dessous lisse, portant au bord externe quelques trichobothridies; carène supérieure antérieure granuleuse. Chez le mâle, la main est plus étroite et plus allongée, la carène digitale, mieux marquée, sépare deux surfaces planes.

Tarses: dernier article des pattes à lobes latéraux arrondis portant 4-5 épines; à son bord inférieur, 4 épines; aucune épine mais des soies au bord externe. Protarsus des pattes I et II avec une frange externe de soies raides, mais sans épine; tarses III et IV sans épine au bord externe inférieur.

Peigne: celui de la femelle est très court et ne porte que 11 à 13 dents, celles-ci ne commençant qu'au tiers de la longueur totale; partie basale à bords légèrement divergents. Le peigne du mâle porte 25 dents; la main de son maxillipède est plus allongée que chez la femelle.

Discussion: c'est de Opisthophthalmus wahlbergi Thor. que cette nouvelle espèce est la plus proche. La conformation des tarses et des protarses des pattes est la même, les sternites sont lisses dans les deux sexes, la vésicule est granuleuse dessous, etc. Mais le peigne est beaucoup plus court puisqu'il ne compte que 11 à 13 dents d'ordinaire, tandis qu'il en montre 15 à 22 dans wahlbergi (17 à 20 d'après Kraepelin). Le céphalothorax est pareil dans les deux sexes, parfaitement lisse dans le triangle interoculaire, un peu granuleux ailleurs, tandis qu'il est différent chez le mâle de $w$. Le bord frontal est profondément échancré dans les deux sexes, "in der Mitte kaum eingeschnitten" (Kraepelin) dans $w$.

De adustus Kraep., il diffère par les sternites tous lisses dans les deux sexes, par la vésicule tuberculeuse dessous; mais il y ressemble par le nombre de dents au peigne et par la réticulation de la surface supérieure de la pince. 


\section{SOLIFUGAE}

Nous connaissons peu de choses concernant les Solifuges d'Angola: Kraepelin, dans le "Tierreich", ne cite qu'une fois le Benguella, pour Solpuga lethalis Koch; dans les résultats de la Mission Rohan Chabot, Louis FAGE mentionne trois espèces: Solpugema (Solpuga) hostilis White, Zeriassa (Zerissa) cuneicornis Purc. et Chelypus macronyx Hewitt. Röwer (Bronns Kl. u. Ordn. des Tierreichs, V, 4, 4, 1934) ajoute encore les espèces suivantes: Mossamedessa abnormis Roewer (Mossamédès), Hemiblossia bouvieri Kraep. (Cours supérieur du Zambèze, dont un tronçon traverse l'Angola), Solpuga venator Pocock (Mossamédès), Solpuga schönlandi Pocock (Cours sup. du Zambèze), Solpuga orthoceras Roewer (Mossamédès), Solpugopa angolensis Roewer (Mossamédès).

La liste des douze espèces de Solifuges connus d'Angola s'établit ainsi :

Chelypus macronyx Hewitt Mossamedessa abnormis Roewer Hemiblossia bouvieri Kraepelin Zeriassa cuneicornis Purcell Solpugassa furcifera Kraepelin Solpuga lethalis Koch(ou rectus Hewitt?) sericea Pocock. venator Pocock. schönlandi Pocock orthoceras Roewer Solpugema hostelis White. Solpugopa angolensis Roewer riv. Kuatiri

Mossamédès cours sup. du Zambèze rive droite du Zambèze Dombondola

Benguella Kakindo, rio Mbalé Mossamédès cours sup. du Zambèze Mossamédès, rio Mbalé riv. Kubya, Luankundu Mossamédès

\section{Collections.}

\section{Solpugassa furcifera Kraep.}

Un exemplaire mâle, venant de Dombondola, à l'extrême Sud de la colonie. L'espèce vient du Damara et de Walfish Bay (Windhuk, Osire, Rehoboth, Herreroland S.); notre trouvaille démontre qu'elle s'étend plus au Nord.

\section{Solpuga sericea Pocock.}

Un mâle et une femelle de Kakindo.

Six mâles et une femelle du Rio Mbalé. 
Ces exemplaires concordent parfaitement à la description qu'en donne Kraepelin; au temps de la parution du "Tierreich " (Lief. 12, 1901), la femelle de cette espèce n'était pas connue.

L'espèce a été décrite du Mashonaland; en outre: Nyassaland. Rhodesia, Congo belge, S.O. africain.

\section{Solpuga orthoceras Röwer.}

Quatre femelles, venant du Rio Mbalé.

L'espèce, découverte à Mossamédès, a été tout récemment décrite par RöweR (1934).

\section{OPILIONIDES}

Les quelques Opilions trouvés ont été déterminés par le Dr Roewer, de Brême. Ils appartiennent à deux espèces, de la famille des Assamiidae.

10 Rhabdopygus fuscus Rwr. 8 đ̊ㅜㅇ, Kalukembé.

Distribution: Gabon, Congo français, Camerun, Congo belge, Guinée portugaise.

$2^{\circ}$ Hypoxestus obscurus Rwr. 3 ơ 오, Ebanga.

Distribution: Est africain, Uganda.

\section{INDEX BIBLIOGRAPHIQUE.}

1. Рососк, R. I.: A Further Revision of the Species belonging to the South-African Genera Uroplectes, Lepreus and Tityolepreus. Ann. Mag. Nat. Hist. (6) 17, 1896, p. 377-393.

2. Kraepelin, K.: Scorpiones und Pedipalpi. Das Tierreich, Lief. 8, 1899.

3. Hinst, S.: On a Collection of Arachnida and Chilopoda ... in Rhodesia. Mem. Proc. Manchester Lit. Phil. Soc., 1911-12, vol. 56, no 2.

4. Krafepelin, K.: Skorpiones und Solifugae. Beitr. zur Kennt. d. Landund Süswasserfauna Deutsch-Südwestafrikas, Lief. 1, 1914, p. 105-136.

5. Hewitr, John.: A Survey of the Scorpion Fauna of South Africa. Trans. Roy. Soc. South Africa, vol. VI, 1917, p. 89-192.

6. Monard, Albert: Matériaux de la Mission scientifique suisse en Angola, Scorpiones. Bull. Soc. neuch. sc. nat., t. 54, 1929, p. 37-43.

7. Kraepelin, Karl: Palpigradi und Solifugae. Das Tierreich, Lief. 12, 1901.

8. Roewer, C. Fr.: Solifugae, Palpigradi, in: Brown's Kl. u. Ordn. des. Tierreichs, V. Bd., IV. Abt., 4. Buch, 1.-5. Lief., 1932-1934. 


\section{$2 \mathrm{BHL}$ Biodiversity Heritage Library}

Monard, A. 1937. "Scorpions, Solifuges et Opilions d'Angola." Revue suisse de zoologie 44, 251-270. https://doi.org/10.5962/bhl.part.117690.

View This Item Online: https://www.biodiversitylibrary.org/item/148528

DOI: $\underline{\text { https://doi.org/10.5962/bhl.part.117690 }}$

Permalink: https://www.biodiversitylibrary.org/partpdf/117690

\section{Holding Institution}

American Museum of Natural History Library

\section{Sponsored by}

BHL-SIL-FEDLINK

\section{Copyright \& Reuse}

Copyright Status: In copyright. Digitized with the permission of the rights holder.

Rights Holder: Muséum d'histoire naturelle - Ville de Genève

This document was created from content at the Biodiversity Heritage Library, the world's largest open access digital library for biodiversity literature and archives. Visit BHL at https://www.biodiversitylibrary.org. 